\title{
ANISOTROPIC PARABOLIC PROBLEMS WITH MEASURES DATA
}

\author{
FARES MOKHTARI
}

Abstract. In this work, we prove the existence of a weak solution of an anisotropic parabolic problem with measure data $u_{t}+A u+F(u, D u)=\mu$ and $u(0)=\mu_{0}$ with $\mu$ and $\mu_{0}$ two Radon bounded measures. The operator $A$ is a Leray-Lions operator with anisotropic growth conditions. Our approach is based on the anisotropic Sobolev inequality, a regularity result, a compactness result, and an integration by parts formula.

Mathematics subject classification (2010): 28C05, 35K10, 35K20, 35K55.

Keywords and phrases: parabolic equation, Radon measure, anisotropic problem.

\section{REFERENCES}

[1] Y. AтIK, T-ensembles locaux et problèmes quasi-linéaires dégénérés à donnée mesure sur un ouvert quelconque, Thèse de Doctorat, Université de Poitiers, 1993.

[2] M. Bendahmane, M. LAnglais, M. SAAD, On some anisotropic reaction-diffusion systems with $L^{1}$-data modeling the propagation of an epidemic disease, Nonlinear Analysis, 54 (2003), 617-636.

[3] M. Bendahmane, Kenneth H. Karlsen, Renormalized entropy solutions for quasi-linear anisotropic degenerate parabolic equations, SIAM J. Math. Anal., 36, 2 (2004), 405-422.

[4] H. BRÉZIS, Opérateurs maximaux monotones et semi-groupes de contractions dans les espaces de Hilbert, North-Holland Publishing company- Amsterdam-London, 1973.

[5] L. Boccardo, T. Gallouët, P. Marcellini, Anisotropic Equations in L ${ }^{1}$, Differential and Integral Equations, 9 (1996), 209-212.

[6] L. Boccardo, A. Dall'Aglio, T. Gallouët, And L. Orsina, Nonlinear Parabolic Equations With Measure Data, Journal of Functional Analysis, 147 (1997), 237-258.

[7] H. BRÉzIS, Analyse Fonctionnelle, théorie et applications, Masson, Paris, 1987.

[8] A. El Hamidi, J. M. Rakotoson, On a perturbed anisotropic equation with a critical exponent, Ricerche di Matematica, 55 (2006), 55-69.

[9] F. LI, Anisotropic Elliptic Equations in $L^{m *}$, Journal of Convex Analysis, 8 (2001), 417-422.

[10] F. Li AND H. ZhaO, Anisotropic parabolic equations with measure data, J. Partial Differ. Equations, 14, 1 (2001), 21-30.

[11] F. LI, Nonlinear degenerate parabolic equations with measure data, Nonlinear Analisis, 61 (2005), 1269-1282.

[12] F. LI, Anisotropic parabolic equations with measure data II, Math. Nachr., 279, 13-14 (2006), 15851596.

[13] I. Fragala, F. Gazzola, B. KAWOHL, Existence and nonexistence results for anisotropic quasilinear elliptic equations, Ann. I. H. Poincaré, 21 (2004), 715-734.

[14] J. L. Lions, Quelques méthodes de résolution des problèmes au limites, Dunod, Paris, 1969.

[15] F. MokHTARI, Etude d'un problème non linéaire du type elliptique-parabolique à données mesures: existence, régularité et comportement de solutions au voisinage des points singuliers, Mémoire de Magister, Ecole Normale Supérieure, Vieux Kouba, Alger, Algérie, 2002.

[16] J. M. RAKOtoson, A compactness lemma for quasilinear problems: application to parabolic equations, J. Funct. Analysis, 106 (1992), 358-374.

[17] J. M. Rakotoson, T-sets and relaxed solutions for parabolic equations, J. Differ. Equations, 111, 2 (1994), 458-471.

[18] J. Simon, Compact sets in the space $L^{p}(0, T ; B)$, Ann. Mat. Pura. App., 146 (1987), 65-96. 
[19] R. Temam, Navier Stokes equations, theory and numerical analysis, North-Holland, Amsterdam, 1984.

[20] R. Temam, Infinite Dimensional Dynamical Sysytems in Mechanics and Physics, Springer-Verlag, 1988.

[21] M. Troisi, Theoremi di inclusione per Spazi di Sobolev non isotropi, Ricerche Mat., 18 (1969), 3-24. 